\title{
Synthesis and structural characterization of Al-CNT metal matrix composite using Physical Mixing Method.
}

\author{
Lubna Rais ${ }^{1}$, Dr. Rajneesh Sharma ${ }^{2}$, Dr. Vimal Sharma ${ }^{3}$ \\ I'Microelectronics, Department of Electronics \&Communication Engineering, Chitkara University, Himachal \\ Pradesh India) \\ ${ }^{2}$ (Department of Electronics \& Communication Engineering, Chitkara University, Himachal Pradesh India) \\ ${ }^{3}$ (Department of Physics, NIT Hamirpur, Himachal Pradesh India)
}

\begin{abstract}
In the present study Carbon nanotube(CNT) reinforced (Al) composite was synthesized by physical mixing method and CNT's distribution within the matrix was traced and characterized. Ultrasonication was used to disperse the CNTs in Al nano powder followed by magnetic stirring. Samples of different weight percentage of $C N T(0.5 w t \%, 1 w t \%, 1.5 w t \%, 2 w t \%$ of $C N T)$ were obtained using this technique. Stuructural characteristics of the samples were explored using X-Ray diffraction(XRD),Scanning Electronic Microscope(SEM) and Transmission Electronic Microscope(TEM) technologies. Uniform distribution of CNTs within the matrix and the strength of metal/CNT interface were confirmed from SEM and TEM images. Along with the distribution of CNTs, XRD also validates the phase composition of the composite.
\end{abstract}

Keywords: Carbon nanotube, , physical mixing method, uniform distribution, structural characteristics.

\section{Introduction}

The composite being used nowadays is synthesized when a strong load carrying material known as reinforcement is embedded in a weaker material, known as matrix. Development of CNT-Metal Matrix Composite (CNT-MMC) is helpful in overcoming the inadequacy of metals and alloys in providing both strength and stiffness to a structure .The strength and ductility is provided by the metal matrix and the strength and/or stiffness is provided by the reinforcement . Metal matrix composites not only provide improved thermal conductivity but low coefficient of thermal expansion making it suitable for electronic applications [1].Although $\mathrm{Al}$ is highly ductile, malleable and low density but cannot be kept in its pure form because it immediately forms oxide layer on the surface when exposed to air, relatively low strength is also a limiting factor [2]. The Al-CNT composite is synthesized in order to keep the desired properties of $\mathrm{Al}$ intact while improving the limiting factors. Reinforcement of Aluminium with variable percentage of carbon nanotubes (CNT) using different methods for the development of lighter weight nano-composite not only improves the homogeneity, reduce the susceptibility to oxidation, but also enhance the strength and stiffness, wear resistance, stable friction coefficient, density and coefficient of thermal expansion which make it suitable for the lightweight industries, such as aerospace, automotive industries etc. [3]

Different methods of synthesizing Al-CNT composite have been used till now which include Powder Metallurgy, Conventional Compaction and Sintering, Hot Press consolidation process, Spark plasma sintering and Powder Rolling etc. In powder metallurgy process, separation of CNTs was affected and CNTs were also damaged during the secondary process [4]. On investigation of interfacial region of Al-CNT composite synthesized using hot pressing and sintering process it was found that $\mathrm{Al}-\mathrm{C}$ reactions occurred at locations containing amorphous carbon, at defect sites and at open ends of CNT. Irregular shaped nanosized carbide particles formed at the end of the CNT which were forming a bond with them as well as the Al beads. At high temperatures, a reaction may occur in $\mathrm{Al}-\mathrm{CNT}$ composite between the $\mathrm{Al}$ and the carbon to form $\mathrm{Al}_{3} \mathrm{C}_{4}$ on the interface taking the shape of needles which reduce the composite strength [5].The need for Al-CNT composite with uniform distribution of CNT along the matrix without or with lesser agglomeration and free from carbides or oxides led to the present synthesis process. In the present work pure Aluminium (99.99\%) nanosized powder is used to synthesize the composite with CNT. The XRD analysis has confirmed absence of any carbide or oxide.

\section{Experimental detail}

The material required for synthesizing AL-CNT metal matrix composite was bought from Nanoshel technologies(panchkula, haryana).The size of granules of Al metal in nano powder is of the order of $80-124 \mathrm{~nm}$ with $99.99 \%$ purity and spherical morphology $\left(\right.$ bulk density: $0.08-0.20 \mathrm{~g} / \mathrm{m}^{3}$ ) while the CNTs of $50 \mathrm{~nm}$ diameter, $3-6 \mu \mathrm{m}$ and $99.99 \%$ purity are used. The solvent used, in which CNTs have to be functionalized, is Ethanol of 99.99\% purity. It was decided to synthesize composites having four different ratios. In all the samples the amount of CNTs was varied while keeping powder quantity constant. 2grams of Aluminium powder was used 
in each sample and compositions with $0.5 \mathrm{wt} \%, 1 \mathrm{wt} \%, 1.5 \mathrm{WT} \%$ and $2 \mathrm{wt} \%$ CNTs were synthesized using physical mixing method.

Both the elements were weighed with utmost accuracy in electronic balance. Before starting the synthesis the CNTs were checked for their settling time in the solvent. It was observed that after one hour of sonication in ethanol the CNTs settled down within five minutes, so the time of sonication was increased. It was observed that CNTS should be sonicated for around six hours to obtain the desired res-*/ult.

In this process firstly, both the elements were sonicated for six hours in ethanol as a solvent in separate beakers in ultrasonicator. After that both the solutions were mixed in one beaker and sonicated for another two hours. Then, obtained solution was reduced to a paste by keeping it on a magnetic stirrer with hotplate for another two hours. The temperature of the hotplate was not more than $80^{\circ} \mathrm{C}$ while stirring speed was constantly varied. The paste was then dried on a hotplate to obtain small pebbles like mixture which was then grinded to powder form. The different powdered compositions were then obtained using the same method. The characterization of the samples was done using XRD analysis, SEM and TEM images. The Bruker D8 X-ray diffractometers was used for the phase analysis with angle range: $5^{\circ}-120^{\circ}$ of composite sample with $2 \mathrm{wt} \%$ of CNT. Images obtained from TEM TECNAI G2 20 S-TWIN with 1000KX magnification, 0.24nm point and $0.14 \mathrm{~nm}$ line resolution and Scanning Electron Microscope: LEO $435 \mathrm{VP}$ with resolution : 4nm in HV and $6 \mathrm{~nm}$ in VP and magnification : 10x to 300,000x

\section{Results and discussion}

The process discussed above can produce homogeneously dispersed CNTs implanted in Al nano powder. The key feature of this process is that the CNTs and nanosized metal particle are mixed homogeneously in an aqueous solution by physical mixing. The critical problem of the strong agglomeration of CNTs reported in other methods within a matrix and strong bonding between CNTs and a matrix can be solved by using the physical mixing process. The CNTs were homogeneously dispersed within grains or at grain boundaries of the Aluminium matrix.[6].This has been observed earlier that metal beads of nanosize align themselves on to the CNTs tubular structure when CNTs are mixed with nano powders of metals like $\mathrm{Cu}, \mathrm{Ag}, \mathrm{Au}$ etc.[7]. In this study, nanopowder has been used while Fig.1 shows the presence of CNT in the composite, it is clear from the SEM image shown in Fig. 2 that Al nano particles have got beautifully embedded on to CNTs tubular structure. The physical effect of shockwave treatment during ultrasonication on CNTs has lead to this uniform dispersion. Before taking the TEM images of the sample, it was sonicated for one hour in an ultrasonicator of frequency 30 $\mathrm{KHz}$. The image shown in Fig. 3 confirms that there is strong bonding between CNTs and nanoparticles, as both are intact even after the shockwave treatment. Although, Al-CNT composite obtained through covalent interactions results in improved properties, but covalent bonding between metal nanoparticles and CNTs is obtained through different acid treatments to create linking groups like, carboxyl $(-\mathrm{COOH})$, carbonyl $(-\mathrm{C}=\mathrm{O})$, and hydroxyl $(-\mathrm{OH})$; this greatly degrades mechanical and electronic transport properties of CNTs as defects are introduced[8] The method followed in this study creates non

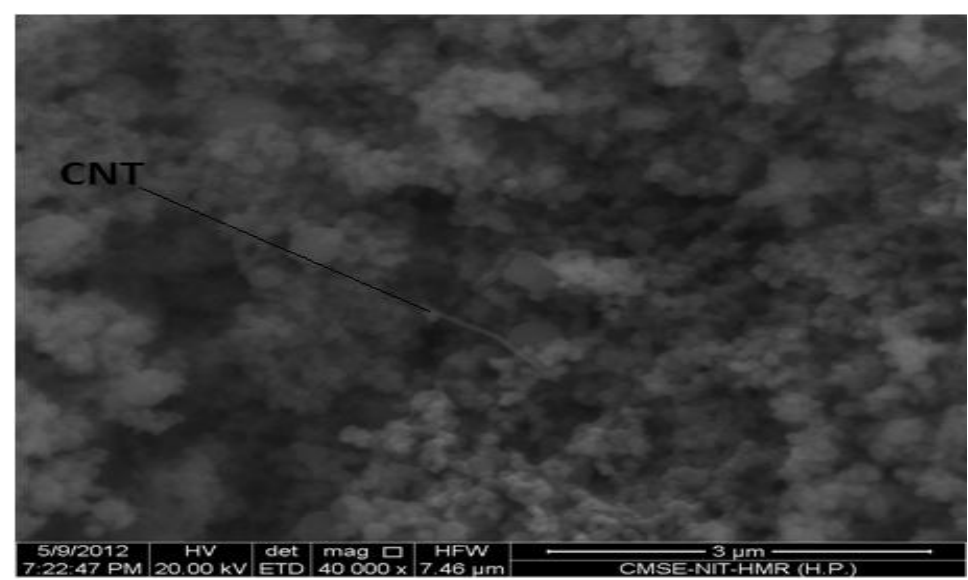

Figure 1 SEM image of the Al-CNT composite showing presence of CNT 


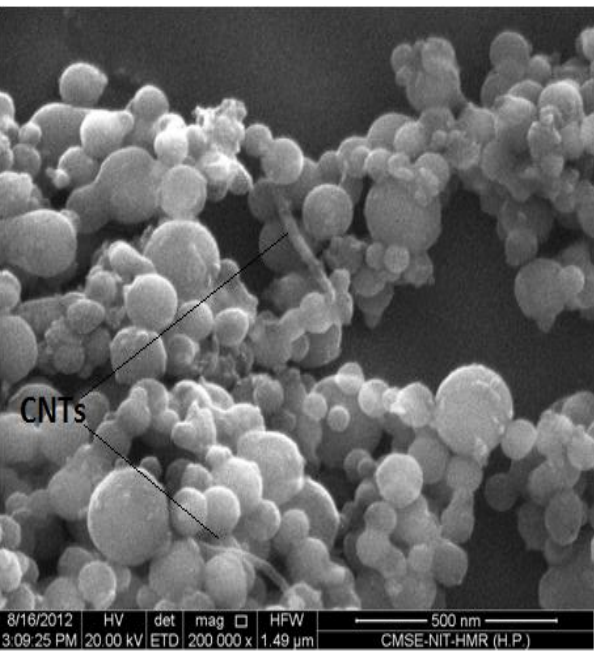

Figure 2SEM image of Al-CNT composite with 2wt\% of CNT

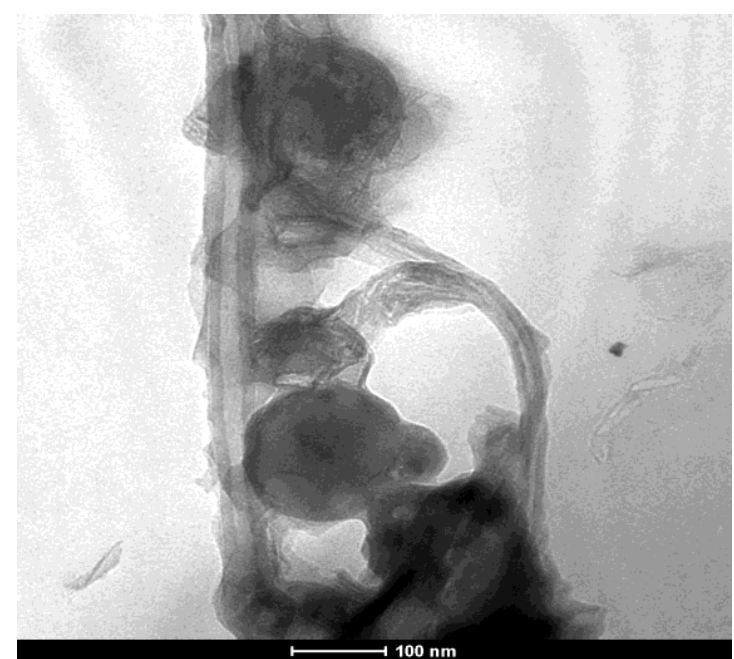

Figure 3 TEM image of Al-CNT composite with $1.5 w t \%$ of CNT

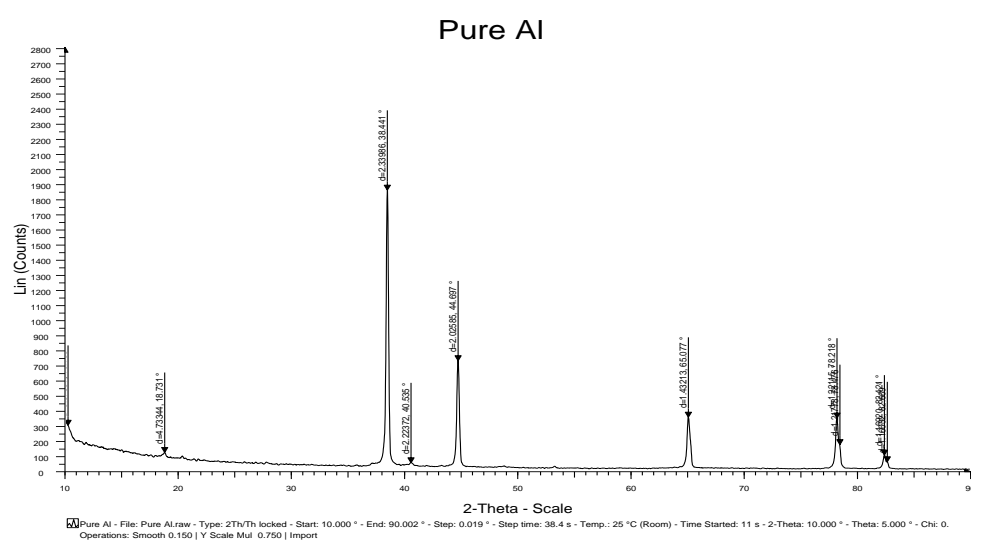

Figure 4 XRD analysis of pure $\mathrm{Al}$ 


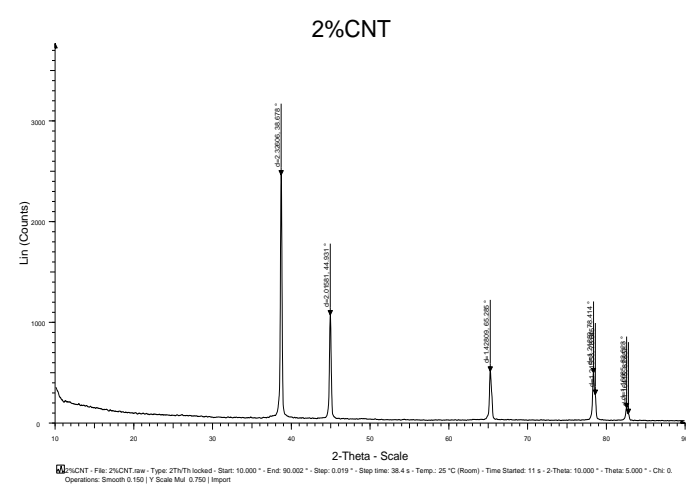

\section{Figure 5 XRD analysis of Al-CNT composite with 2wt\% of CNT}

Covalent bonding between the two materials, resulting in less defects and enhanced properties. The XRD analysis of pure and Al-CNT composite containing 2wt\% of CNT was done and is as shown ,respectively, in the fig. [4 \& 5] XRD is done to investigate the presence of CNT peaks which is used for phase analysis. It's clear from the fig that no new peak has been introduced due to presence of CNT. Although no carbon peaks are identified in XRD analysis but TEM images in Fig.3 clearly indicate the presence of CNTs. The absence of any new peak due to CNTs may be due to uniform distribution of CNTs over matrix because peak appears only when CNTs are clustered and diminishes if CNTs are well dispersed. It may also happen because of small amount of CNTs used or amorphization of CNTs

\section{Conclusion}

The synthesis of a CNT- nanoparticle composite by ultrasonication was reported. Dispersion of CNTs and synthesis of a nanoparticles were possible by combination ultrasonic treatment and use of organic dispersant , ethanol. It's as a good dispersant when sonication is used to functionalize the nanoparticles. The characterizations techniques used confirmed the formation of a strong bonding between $\mathrm{Al}$ and CNT without any phase change.

\section{REFERENCES}

[1] Karl U. Kainer, Metal Matrix Composites. Custom-made Materials for Automotive and Aerospace Engineering( Weinheim,WILEYVCH Verlag GmbH \& Co. KGaA, 2006)

[2] Umma A, Maleque M.A, Iskandar I.Y and Mohammed Y.A Umma A, Carbon Nano tube Reinforced Matrix Nano-Composite: a Critical Review, Australian Journal of Basic and Applied Sciences, 6(12) 2012, 69-75

[3] M K SURAPPA matrix composites: Challenges and Opportunities, S $^{-}$adhan $^{-}$a, Vol. $28(1$ \& 2) 2003, 319-320.

[4] S. R. Bakshi, D. Lahiri and A. Agarwal, Carbon nanotube reinforced metal matrix composites - a review, International Materials Reviews, vol55(1) 2010,42-50.

[5] M.S. Senthil Saravanan, S.P. Kumaresh Babu*, K. Sivaprasad, Mechanically Alloyed Carbon Nanotubes (CNT) Reinforced NanocrystallineAA 4032: Synthesis and Characterization, Journal of Minerals \& Materials Characterization \& Engineering, Vol. 9(11) 2010, 1032-1033.

[6] Jinzhi Liao, Ming-Jen Tan, Raju V. Ramanuja, Shashwat Shukla,Carbon nanotube evolution in Aluminium matrix during composite fabrication process, Materials Science Forum, Vol. 690 ,2011, 294-297.

[7] Junhong Chen and Ganhua Lu , Carbon Nanotube-Nanoparticle Hybrid Structures, in Jose Mauricio Marulanda(Ed.),Carbon Nanotubes 31 (China, InTech China,2010) 612-618.

[8] Junhong Chen and Ganhua Lu , Carbon Nanotube-Nanoparticle Hybrid Structures, in Jose Mauricio Marulanda(Ed.),Carbon Nanotubes 31 (China, InTech China,2010) 623-634 